\title{
Toaster oven helps researchers toy with microfluidics
}

As a founding faculty member at a brand-new university, Michelle Khine faced a problem in getting started on her work. Trained as an engineer, she planned to build her research around the development of microfluidic devices, the popular 'lab on a chip' systems that consist of channels, pumps and cellular growth chambers arranged together on tiny silicon wafers. Unfortunately, when she arrived to start her job in July 2006, the clean room required to make these devices hadn't been built yet.

"We had no facilities whatsoever; these things take a long time, and I'm not very patient," says Khine, a professor of engineering at the newly established University of California, Merced.

Desperate to start shrinking some of her new designs down to the appropriate chip size, she had a sudden inspiration. "I remembered my favorite childhood toy, when I was six years old," she says, referring to special plastic sheets called 'Shrinky-Dinks' that children can cut and decorate. When heated, the plastic shrinks uniformly, making perfect miniatures of the two-dimensional figures.

Khine used a standard laser printer to precisely copy one of her chip designs from her computer onto Shrinky-Dinks plastic. She then cooked the sheet in a toaster oven at $160{ }^{\circ} \mathrm{C}$ for about ten minutes. Not only did the design shrink by $63 \%$, but the areas with ink on them bulged upward, creating perfectly defined ridges and channels that work just like those carefully etched onto standard microfluidic features. "It took me 25 years, but I finally found a use for Shrinky-Dinks," Khine says.

Replacing hundreds of thousands of dollars' worth of specialized equipment with a printer and a toaster oven, the new technique brings microfluidic devices within reach of any labor kitchen. The Shrinky-Dinks approach also saves time, allowing researchers to build and test prototype chips in a few hours instead of weeks.

Even with its advantages, the new method won't put conventional chip builders out of business. Among other limitations, Khine's technique cannot produce features smaller than $70 \mu \mathrm{m}$ across, or about the diameter of a thick human hair, whereas conventional photolithography achieves $2-\mu \mathrm{m}$ resolution. However, most microfluidic devices used in biological research contain channels and chambers $100 \mu \mathrm{m}$ wide or larger, making them perfect candidates for the Shrinky-Dinks approach.

Indeed, Khine's first paper on the technique, published online last November, has drawn enormous interest from biologists ( $L a b$
Chip 8, 170-172; 2008). "I couldn't believe the response that we got. Everyone all over the world was emailing me," says Khine. According to the journal's publisher, the paper has received more downloads than any other article they have published.

Kara McCloskey, another professor of engineering at UC Merced, is one of the new Shrinky-Dinks fans. In her research on stem cell differentiation, McCloskey needs to culture developing clusters of embryonic stem cells. The standard technique for doing that, called 'hanging-drop culture', involves studying cells in drops of fluid on glass and, for that reason, is labor intensive and error prone. "Every time you want to control the growth factors, you're literally pipetting the media in and out," says McCloskey.

Now, she and her colleagues simply manipulate the cells on specially designed Shrinky-Dinks devices. The cells fit into wells on the plastic, and channels connected to the wells deliver precise doses of different media.

Khine, meanwhile, is now busy designing a second generation of Shrinky-Dinks-inspired chips that rely on microscopic electrical circuits and pumps to control conditions in each tiny well. It's a small world, after all, at least when it comes to microfluidic devices.

Alan Dove, Springfield, Massachusetts

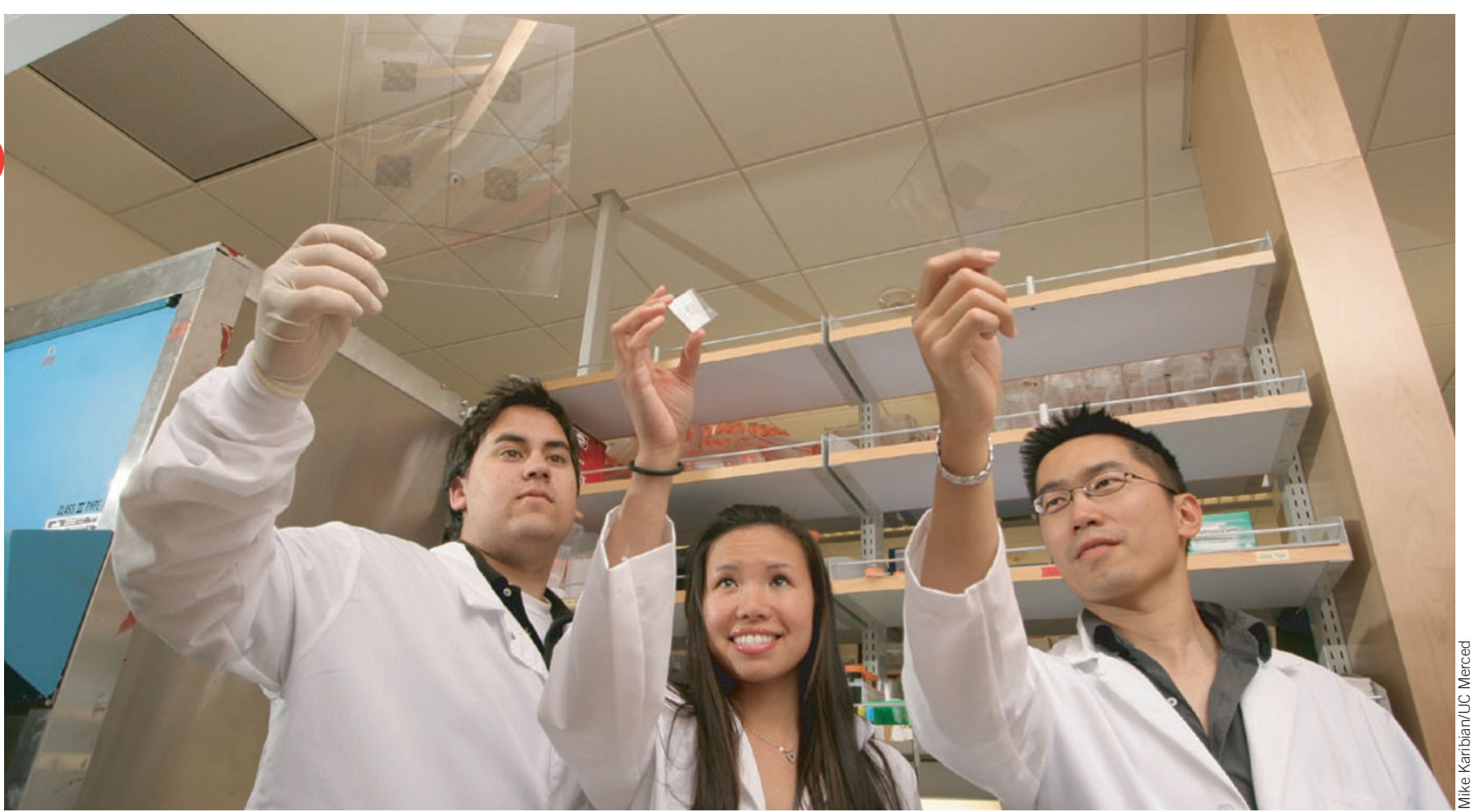

Printing a solution: Michelle Khine (center) and her students Anthony Grimes (left) and Chi-Shuo Chen (right), who helped develop the approach 